KREUZUNGSPARAMETER FÜR EIGENSCHAFTEN DER MILCHLEISTLNG BEI DER RASSENKREUZUNG SIMMENTAL X RED -HOLSTEIN

\title{
J. SCHMIDLIN
}

Institut für Tierproduktion, Gruppe Ticrzucht, Eidg. Technische Hochschule, Zïrich, Schweiz

Seit I 967 wird in der Schweiz Sperma von Red-Holstein Stieren beim Simmentalev Fleckvieh eingesetzt. Untersucht werden die Auswirkungen der Einkreuzung auf die Milchleistung.

Mit steigendem Red-Holstein blutanteil geht eine starke Erhöhung der Milchleistung, aber ein Rückgang des Milchgehaltes einher. Die Kreuzungsparameter für die Milchleistung in der I. Laktation (305 Tage) betragen $1387 \mathrm{~kg}$ für die Rasse-differenz, $282 \mathrm{~kg}$ für die Heterosis und $-3^{83} \mathrm{~kg}$ für die Rekombination.

DIE KONSERVIERUNG DER GAMETEN EINIGER SÜBWASSERFISCHE

\section{H. STEIN}

TU München/Weihenstephan, Institut $f$. Tierwissenschaften, Angewandte Zoologic, 8050 Freising-Weihenstephan (FRG)

Die Kurzzeitkonservierung von Fischsperma war bei $4^{\circ} \mathrm{C} 24-3^{6}$ Stunden möglich. Die Gefrierkonservierung von Salmoniden- und Hechtsperma wurde unter Verwendung der Pellettechnik nach Nagase (1964) erprobt. Die durchschnitliche Befruchtungsrate war nach dem Auftauen bei Salma gairdneri, Salmo trutta forma fario und Salmo trutta forma lacustris 80 p. Ioo. Die Resultate bei den anderen Arten waren sehr unterschiedlich. Gefrierkonservierte Eier konnten in keinem Fall befruchtet werden. Die Kurzzeitkonservierung ergab über roo Stunden nahezu gleichbleibende Resultate.

MAST- UND SCHLACHTLEISTUNGSVERGLEICH BEI KÄLBERN UND JUNGSTIEREN VON BRAUNVIEH

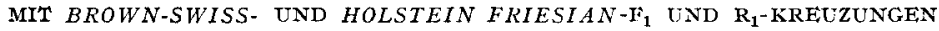

\section{R. STEINWENDER}

Bundesversuchsanstalt $f$. alpenländische Landwirtschaft Gumpcnstein, A-8952 Irdning, Abteilung Tierproduktion (Österreich)

Mit allen Stierkälbern aus einem laufenden Verdrängungskreuzungsversuch wurde an der Bundesversuchsanstalt Gumpenstein mit I34 Mastkälbern und mit 147 Jungmaststieren ein Mastleistungs- und Schlachtkörperwertvergleich durchgeführt. Die Tiere verteilen sich in der $\mathrm{F}_{1}$-Generation (50 p. Ioo Fremdgenanteil) auf die 3 Gruppen BV, BV $\times$ BS und $\mathrm{BV} \times \mathrm{HF}$ und in der $\mathrm{R}_{1}$-Generation (ca. 75 p. Ioo Fremdgenanteil) auf die 4 Gruppen $\mathrm{BV},(\mathrm{BV} \times \mathrm{BS}) \times \mathrm{BS}$, $(\mathrm{BV} \times \mathrm{HF}) \times \mathrm{HF}$ und $(\mathrm{DS} \times \mathrm{HF}) \times \mathrm{HF}$.

Im Rahmen der $\mathrm{K}$ älbermast war in der $\mathrm{F}_{1}$-Generation die $Z$ weihälftenausbeute der $\mathrm{BV} \times \mathrm{BS}-$ Gruppe signifikant $(P \leqslant 0.05)$ schlechter und der Keulen- und Fleischanteil vom BV signifikant höher.

In der $R_{\mathbf{1}}$-Generation ergaben sich signifikante Unterschiede in der Futterverwertung und $Z$ weihälftenausbeute. Die Teilstückzerlegung ergab den höchsten Brustanteil beim BV und niedrigere Keulenanteile der Gruppen $\mathrm{DS} \times \mathrm{HF}(\mathrm{P} \leqslant 0.0 \mathrm{I})$ und $\mathrm{BV} \times \mathrm{HF}(\mathrm{P} \leqslant 0.05)$. Beim Nierenfett hatte die BV-Gruppe die niedrigsten und die DS $\times$ HF-Gruppe die höchsten Werte. In der Keule hatte das BV den höchsten Fleisch- und niedrigsten Knochenanteil.

In der Jungstiermast zeigte die $\mathrm{F}_{1}$-Generation beim BV einen wesentlich höheren Fleischund niedrigeren Knochenanteil, der Anteil wertvoller Teilstücke war nur tendenziell höher. Die $R_{1}$-Generation wies in der BV-und BV $\times$ BS-Gruppe einen höeren Fleisch-anteil $(\mathrm{P} \leqslant 0.0 \mathrm{I})$ und niedrigeren Fett- sowie beim BV auch Knochen-anteil auf. Im Anteil wertvoller Teilstiicke lag die $B V$ und $B V \times$ BS Gruppe signifikant $(P \leqslant 0.01)$ iiber den beiden anderen Gruppen. In den übrigen Merkmalen waren keine gesicherten Unterschiede nachweisbar. 